Canad. Math. Bull. Vol. 23 (2), 1980

\title{
A GENERALIZATION OF THE LAX-MILGRAM LEMMA
}

\author{
BY \\ K. INAYATNOOR AND M. ASLAM NOOR
}

Let $H$ be a real Hilbert space with its dual space $H^{\prime}$. The norm and inner product in $H$ are denoted by $\|\cdot\|$ and $\langle.,$.$\rangle respectively. We denote by \langle.,$.$\rangle , the$ pairing between $H^{\prime}$ and $H$.

If $a(u, v)$ is a bilinear form and $F$ is a real-valued contınuous functional on $H$, then we consider $I[v]$, a functional defined by

$$
I[v]=a(v, v)-2 F(v), \quad \text { for all } \quad v \in H .
$$

It has been shown by Noor and Whiteman [5], that under certain conditions on $a(u, v)$ and $F$, the minimum of $I(v)$ on $H$ can be characterized by

$$
a(u, v)=\left\langle F^{\prime}(u), v\right\rangle, \quad \text { for all } \quad v \in H,
$$

where $F^{\prime}(u)$ is the Fréchet derivative of $F$ at $u \in H$.

For a linear continuous functional $F$, solving equation (1) is equivalent to finding $u \in H$ such that

$$
a(u, v)=\langle F, v\rangle, \quad \text { for all } \quad v \in H,
$$

and this is the well known Lax-Milgram lemma [2].

The motivation of this paper is to show that under certain conditions, there does exist a unique solution of a more general equation of which (1) is a special case. Our result can be considered as a representation theorem analogous to the Lax-Milgram lemma for a class of nonlinear problems.

Definition 1. The operator $T: H \rightarrow H^{\prime}$ is called antimonotone, if

$$
\langle T u-T v, u-v\rangle \leq 0, \quad \text { for all } u, v \in H,
$$

and is said to be Lipschitz continuous, if there exists a constant $\gamma>0$ such that

$$
\|T u-T v\| \leq \gamma\|u-v\|, \quad \text { for all } u, v \in H .
$$

Definition 2. A bilinear form $a(u, v)$ on $H$ is said to be coercive [3] and continuous, if there exist constants $\rho>0, \mu>0$ such that

$$
a(v, v) \geq \rho\|v\|^{2}, \quad \text { for all } v \in H,
$$

Received by the editors October 25, 1978. 
and

$$
|a(u, v)| \leq \mu\|u\|\|v\|, \quad \text { for all } \quad u, v \in H .
$$

In particular, it follows that $\rho \leq \mu$, see [4]. If $a(u, v)$ is continuous coercive bilinear form, then by the Riesz-Fréchet representation theorem [1], we have

$$
a(u, v)=\langle T u, v\rangle, \quad \text { for all } \quad v \in H .
$$

It has been shown [4] that $\|T\| \leq \mu$. Finally, we define $\Lambda$, a canonical isomorphism from $H^{\prime}$ onto $H$ by

$$
\langle f, v\rangle=(\Lambda f, v), \quad \text { for all } \quad v \in H, f \in H^{\prime} .
$$

Then $\|\Lambda\|_{H^{\prime}}=\left\|\Lambda^{-1}\right\|_{H}=1$.

We make the following hypothesis.

Condition $N$. We assume that $\gamma<\rho$, where $\gamma$ is the Lipschitz constant of the nonlinear operator $A$ and $\rho$ is the coercivity constant.

We now state and prove the main result.

THEOREM 1. Let $a(u, v)$ be a coercive continuous bilinear form and $A$ is a Lipschitz continuous antimonotone operator. If condition $N$ holds, then there exists a unique $u \in H$ such that

$$
a(u, v)=\langle A(u), v\rangle, \quad \text { for all } \quad v \in H .
$$

Moreover, if $a(u, v)$ is a symmetric positive bilinear form and $A(u)=F^{\prime}(u)$, the Fréchet derivative of $F$ at $u$, then solving (3) is equivalent to finding $\operatorname{Min}_{v \in \boldsymbol{H}}\{a(v, v)-2 F(v)\}$, as shown in [5].

We need the following lemma, which is essentially due to Noor [4]. We include its proof for the sake of completeness.

LEMmA 1. Let $\xi$ be a number such that $0<\xi<2\left(\rho-\gamma / \mu^{2}-\gamma^{2}\right)$ and $\gamma \xi<1$. Then there exists a $\theta$ with $0<\theta<1$ such that

$$
\left\|\Phi\left(u_{1}\right)-\Phi\left(u_{2}\right)\right\| \leq \theta\left\|u_{1}-u_{2}\right\|, \quad \text { for all } u_{1}, u_{2} \in H,
$$

where for $u \in H, \Phi(u) \in H^{\prime}$ is defined by

$$
\langle\Phi(u), v\rangle=(u, v)-\xi a(u, v)+\xi\langle A(u), v\rangle, \quad \text { for all } \quad v \in H .
$$

Proof. For all $u_{1}, u_{2} \in H$,

$$
\begin{gathered}
\left\langle\Phi\left(u_{1}\right)-\Phi\left(u_{2}\right), v\right\rangle=\left(u_{1}-u_{2}, v\right)-\xi a\left(u_{1}-u_{2}, v\right)+\xi\left\langle A\left(u_{i}\right)-A\left(u_{2}\right), v\right\rangle, \\
\quad \text { for all } v \in H . \\
=\left(u_{1}-u_{2}, v\right)-\xi\left\langle T\left(u_{1}-u_{2}\right), v\right\rangle+\xi\left\langle A\left(u_{1}\right)-A\left(u_{2}\right), v\right\rangle \\
=\left(u_{1}-u_{2}, v\right)-\xi\left(\Lambda T\left(u_{1}-u_{2}\right), v\right)+\xi\left(\Lambda A\left(u_{1}\right)-\Lambda A\left(u_{2}\right), v\right), \text { by }(2) . \\
=\left(u_{1}-u_{2}-\xi \Lambda T\left(u_{1}-u_{2}\right), v\right)+\xi\left(\Lambda A\left(u_{1}\right)-\Lambda A\left(u_{2}\right), v\right) .
\end{gathered}
$$


Thus

$$
\left|\left\langle\Phi\left(u_{1}\right)-\Phi\left(u_{2}\right), v\right\rangle\right| \leq\left\|u_{1}-u_{2}-\xi \Lambda T\left(u_{1}-u_{2}\right)\right\|\|v\|+\xi\left\|A\left(u_{1}\right)-A\left(u_{2}\right)\right\|\|v\| .
$$

Now by $\|T\| \leq \mu$ and the coercivity of $a(u, v)$, it follows that

$$
\begin{aligned}
\left\|u_{1}-u_{2}-\xi \Lambda T\left(u_{1}-u_{2}\right)\right\|^{2} & \leq\left\|u_{1}-u_{2}\right\|^{2}+\xi^{2}\|T\|^{2}\left\|u_{1}-u_{2}\right\|^{2}-2 \xi a\left(u_{1}-u_{2}, u_{1}-u_{2}\right), \\
& \leq\left(1+\xi^{2} \mu^{2}-2 \xi \rho\right)\left\|u_{1}-u_{2}\right\|^{2} .
\end{aligned}
$$

Hence

$$
\begin{aligned}
\left|\left\langle\Phi\left(u_{1}\right)-\Phi\left(u_{2}\right), v\right\rangle\right| & \leq\left(\sqrt{ } 1+\xi^{2} \mu^{2}-2 \xi \rho\right)\left\|u_{1}-u_{2}\right\|\|v\|+\xi\left\|A\left(u_{1}\right)-A\left(u_{2}\right)\right\|\|v\|, \\
& \leq\left\{\left(\sqrt{ } 1+\xi^{2} \mu^{2}-2 \xi \rho\right)+\xi \gamma\right\}\left\|u_{1}-u_{2}\right\|\|v\|,
\end{aligned}
$$

by the Lipschitz continuity of $A$.

$$
=\theta\left\|u_{1}-u_{2}\right\|\|v\|,
$$

where $\theta=\sqrt{ } 1+\xi^{2} \mu^{2}-2 \xi \rho+\gamma \xi<1$ for $0<\xi<2\left(\rho-\gamma / \mu^{2}-\gamma^{2}\right)$, and $\xi \gamma<1$, because $\gamma<\rho$ by condition $N$.

Thus for all $u_{1}, u_{2} \in H$,

$$
\begin{aligned}
\left\|\Phi\left(u_{1}\right)-\Phi\left(u_{2}\right)\right\|_{H^{\prime}} & =\sup _{v \in \mathbf{H}} \frac{\left|\left\langle\Phi\left(u_{1}\right)-\Phi\left(u_{2}\right), v\right\rangle\right|}{\|v\|} \\
& \leq \theta\left\|u_{1}-u_{2}\right\| .
\end{aligned}
$$

\section{Proof of theorem 1. Uniqueness.}

Let $u_{1}, u_{2}$ be two solutions in $H$ of

$$
\begin{aligned}
& a\left(u_{1}, v\right)=\left\langle A\left(u_{1}\right), v\right\rangle \quad \text { for all } v \in H, \\
& a\left(u_{2}, v\right)=\left\langle A\left(u_{2}\right), v\right\rangle \quad \text { for all } v \in H \text {. }
\end{aligned}
$$

Thus by subtracting and taking $v$ as $\left(u_{1}-u_{2}\right)$, we get

$$
a\left(u_{1}-u_{2}, u_{1}-u_{2}\right)=\left\langle A\left(u_{1}\right)-A\left(u_{2}\right), u_{1}-u_{2}\right\rangle .
$$

By the coercivity of $a(u, v)$ and the antimonotonicity of $A$, it follows that there exists $\rho>0$ such that

$$
\begin{aligned}
\rho\left\|u_{1}-u_{2}\right\|^{2} & \leq a\left(u_{1}-u_{2}, u_{1}-u_{2}\right) \\
& =\left\langle A\left(u_{1}\right)-A\left(u_{2}\right), u_{1}-u_{2}\right\rangle \\
& \leq 0
\end{aligned}
$$

Hence $u_{1}=u_{2}$, the uniqueness.

Existence. For a fixed $\xi$ as in lemma 1 and $u \in H$, define $\Phi(u) \in H^{\prime}$, by (4). Thus by the Riesz-Fréchet theorem, there exists a unique $w \in H$ such that

$$
(w, v)=\langle\Phi(u), v\rangle \quad \text { for all } \quad v \in H,
$$


and $w$ is given by

$$
w=\Lambda \Phi(u)=T u,
$$

which defines a map from $H$ into itself.

Now for all $u_{1}, u_{2} \in H$,

$$
\begin{aligned}
\left\|T u_{1}-T u_{2}\right\| & =\left\|\Lambda \Phi\left(u_{1}\right)-\Lambda \Phi\left(u_{2}\right)\right\| \\
& \leq\left\|\Phi\left(u_{1}\right)-\Phi\left(u_{2}\right)\right\| \\
& \leq \theta\left\|u_{1}-u_{2}\right\|, \text { by lemma } 1 .
\end{aligned}
$$

Since $\theta<1, T u$ is a contraction and has a fixed point $T u=u \in H$, which satisfies

$$
\begin{aligned}
(u, v) & =\langle\Phi(u), v\rangle \\
& =(u, v)-\xi a(u, v)+\xi\langle A(u), v\rangle
\end{aligned}
$$

Thus for $\xi>0$, we have

$$
a(u, v)=\langle A(u), v\rangle \quad \text { for all } \quad v \in H .
$$

Remark 1. It is obvious that for $A(u)=F^{1}(u)$, the existence of a unique solution of (1) follows under the assumptions of theorem 1.

If $A$ is independent of $u$, i.e., $A u=f$ (say), then the Lipschitz constant $\gamma$ is zero. Consequently theorm 1 is exactly the same as one proved by Lax and Milgram [2].

Furthermore, for the special case $a(u, v)=(u, v)$, theorem 1 reduces to:

THeOREM 2. If $A$ is Lipschitz continuous antimonotone operator with Lipschitz constant $\gamma<1$, then there exists a unique solution $u \in H$ such that

$$
(u, v)=\langle A(u), v\rangle \quad \text { for all } \quad v \in H .
$$

Theorem 2 shows that the Riesz-Fréchet theorem also holds for a class of monotone operators on $H$, which includes the Fréchet derivatives of nonlinear functionals as a special case.

We give another proof of theorem 1 based on the iteration scheme similar to Picard's and also derive a bound for the error.

We define the iteration $u_{n}$ by the following scheme

$$
a\left(u_{n+1}, v\right)=\left\langle A\left(u_{n}\right), v\right\rangle \quad \text { for all } \quad v \in H .
$$

Theorem 3. If $a(u, v)$ is a positive definite bilinear form on $H$ and $A$ is a Lipschitz continuous operator such that condition $N$ holds, then the iteration $u_{n}$ defined by (5) converges strongly to $u$, the solution of (3) in H. Moreover, the bound for the error, for any $u_{0} \in H$, is given by

$$
\left\|u_{n}-u\right\| \leq \frac{\alpha^{n}}{1-\alpha}\left\|u_{1}-u_{0}\right\|, \quad \text { for } \quad n=0,1,2, \ldots
$$

where $\alpha=\gamma / \rho$. 
Proof. By the coercivity (positive definiteness) of $a(u, v)$, it follows that

$$
\begin{aligned}
\rho\left\|u_{n+1}-u_{n}\right\|^{2} & \leq a\left(u_{n+1}-u_{n}, u_{n+1}-u_{n}\right) \\
& =\left\langle A\left(u_{n}\right)-A\left(u_{n-1}\right), u_{n+1}-u_{n}\right\rangle, \text { by }(5) . \\
& \leq\left\|A\left(u_{n}\right)-A\left(u_{n-1}\right)\right\|,
\end{aligned}
$$

by the Cauchy-Schwarz inequality.

$$
\leq \gamma\left\|u_{n}-u_{n-1}\right\|\left\|u_{n+1}-u_{n}\right\|
$$

by the Lipschitz continuity of $A$.

Thus

$$
\begin{aligned}
\left\|u_{n+1}-u_{n}\right\| & \leq \frac{\gamma}{\rho}\left\|u_{n}-u_{n-1}\right\| \\
& =\alpha\left\|u_{n}-u_{n-1}\right\|,
\end{aligned}
$$

where $\alpha=\gamma / \rho<1$ by condition $N$.

Continuing in this way, we obtain

$$
\left\|u_{n+1}-u_{n}\right\| \leq \alpha^{n}\left\|u_{1}-u_{0}\right\| .
$$

Hence, by the repeated use of the triangle inequality, it follows that

$$
\begin{aligned}
\left\|u_{n+k}-u_{n}\right\| & \leq\left(\alpha^{n+k-1}+\cdots+\alpha^{n}\right)\left\|u_{1}-u_{0}\right\|, \\
& \leq \frac{\alpha^{n}}{1-\alpha}\left\|u_{1}-u_{0}\right\| .
\end{aligned}
$$

Since $\alpha<1$, it follows that $u_{n}$ is a Cauchy sequence and has a limit point such that $u_{n} \rightarrow u \in H$, the unique solution of (3). Also at the same time it implies that

$$
u_{n} \rightarrow u \text { in } H \text { strongly. }
$$

Remark 2. Theorem 3 holds for any general complete normed space. Note that it also shows the existence of a unique solution of (3).

REMARK 3. We note that if $a(u, v)$ is a positive definite bilinear form on $H$, then from (1), it follows that for all $u \in H$,

$$
\begin{aligned}
\rho\|u\|^{2} & \leq a(u, u)=\left\langle F^{\prime}(u), u\right\rangle \\
& \leq\left\|F^{\prime}(u)\right\|_{H^{\prime}}\|u\|,
\end{aligned}
$$

by the Cauchy-Schwarz inequality.

Thus

$$
\|u\| \leq \frac{1}{\rho}\left\|F^{\prime}(u)\right\|_{H^{\prime}}
$$


This expresses the continuous dependence of $u$ on the Fréchet derivative $F^{\prime}(u)$. For the linear functional $F$, it follows that

$$
\|u\| \leq \frac{1}{\rho}\|F\|_{H^{\prime}}
$$

a well known result, see Strang and Fix [6, page 16].

\title{
REFERENCES
}

1. L. Bers, F. John and M. Schechter, Partial differential equations, Academic Press, New York, 1966.

2. P. D. Lax and A. N. Milgram, Parabolic equations, Annals of Math. Study No. 33, Princeton, N.J., (1954), 167-190.

3. J. Lions and G. Stampacchia, Variational inequalities, Comm. Pure Apl. Math., 20 (1967), 493-518.

4. M. Aslam Noor, Variational inequalities and approximation, TR/37, Mathematics Department, Brunel University, 1974.

5. —, and J. R. Whiteman, Error bounds for finite element solutions of mildly nonlinear elliptic boundary value problems, Num. Math. 26 (1976), 107-116.

6. G. Strang and G. Fix, An analysis of the finite element method, Prentice-Hall Inc., Englewood Cliff, N.J. 1973.

\author{
Mathematics Department \\ KERMAN UNIVERSITY \\ KERMAN, IRAN
}

